\title{
L'ÎLE DE LA RÉUNION PENDANT LA GRANDE GUERRE : PATRIOTISME COLONIAL, IDENTIFICATION À LA NATION ET STIGMATISATION DES ENNEMIS
}

Je n'avais pas encore onze ans lorsque survint la "Grande Guerre » de 1914. J'avais appris à l'école que la France avait hâte de se venger de sa défaite de 1870-1871. Nous avions lu «La Dernière Classe de français » d'Alphonse Daudet dans ses Contes du lundi. Nous savions que le traité de Francfort de 1871 avait enlevé à la France l'Alsace, moins Belfort, et une bonne partie de la Lorraine. On parlait encore avec étonnement, voire amusement et ironie, de cette "Entente cordiale " qui reléguait au chapitre des souvenirs l'hostilité franco-britannique, si lourde de conséquences dans notre région du monde ${ }^{1}$.

C'est par ces mots que cet enseignant et militant des droits de l'homme se rappelle les tensions générées par les concurrences internationales et impériales depuis la défaite de 1870 . Fortement mobilisée par l'école ${ }^{2}$ et une presse d'opinion en cours de structuration ${ }^{3}$, la population réunionnaise se sent immédiatement investie d'une mission patriotique. La mobilisation scelle le destin de l'île à celui de sa métropole malgré les difficultés à appliquer un service militaire pérenne et efficace ${ }^{4}$. Les fièvres patriotiques suscitées par l'entrée en guerre laissent ainsi espérer une participation active aux réalités du conflit. Pourtant, la lenteur de la mobilisation et les diff1cultés engendrées par l'éloignement de l'île transforment les logiques de

1. Simon Lucas, Un petit créole et ses souvenirs, Saint-Denis, Océan éditions, 1992, p. 15.

2. Pierre-Éric Fageol, «Le patriotisme au lycée de Saint-Denis de La Réunion avant la Grande Guerre (1870-1914) ", Histoire de l'éducation n 133, janvier-mars 2012, pp. 43-64.

3. Les câblogrammes diffusés dans la presse informent scrupuleusement la population sur la détérioration progressive des relations internationales depuis l'attentat de Sarajevo le 28 juin 1914.

4. Que ce soit en 1872, 1889, 1905 ou 1913, les tentatives pour appliquer un service national à La Réunion se sont soldées par un échec. Les espoirs suscités par l'application d'une loi militaire sont très vite déçus lorsqu'il s'agit de procéder au recensement des populations concernées et à l'examen de leur état de santé. À la veille de la Grande Guerre, un certain nombre de rapports rendent compte des difficultés rencontrées pour enrôler un contingent de qualité à La Réunion. Outre le fait que les autorités aient à déplorer un absentéisme important lors des conseils de révision, les pathologies observées témoignent d'une situation sanitaire inquiétante. Le corps de la Nation ne peut accepter des corps malingres dont le phénotype diffère de celui des « Français de France ». Le pas est rapidement franchi pour dénoncer une "dégénérescence de la race créole » et, pour notre propos, son incapacité à intégrer le giron national. 
participation en déviant la vindicte populaire sur des ennemis intérieurs et une espionnite peu en phase avec la réalité. L'imaginaire patriotique se nourrit alors de fièvres obsidionales permettant aux Réunionnais de se sentir investis d'une mission participative au salut national.

\section{ENTRÉE EN GUERRE ET FIÈVRE PATRIOTIQUE}

Dès l'annonce de la guerre et de la mobilisation le 2 août 1914, les déclarations patriotiques se multiplient et instaurent un climat empreint de gravité mais aussi d'enthousiasme pour sauver la mère Patrie. Rachel Mnémosyne souligne :

Les clairons se font entendre, les gens sortent dans les rues chantant la perte des Allemands, annonçant d'ores et déjà l'enfoncement des fronts par les Français et la reprise de l'Alsace et de la Lorraine. L'Allemagne n'a qu'à bien se tenir ${ }^{5}$.

Même si la peur envahit parfois les esprits, les Réunionnais montrent une certaine résignation face au devoir qui leur incombe. En filigrane se profile également cette idée que la participation à l'effort de guerre peut aboutir à une reconnaissance de la part de la métropole. La participation à la guerre n'est pas considérée seulement comme une réponse à un devoir civique mais bel et bien comme une marque d'union avec le reste de la Nation. Cet état d'esprit est d'autant plus partagé que le conflit est considéré comme juste puisqu'il résulte d'une agression de la part d'un ennemi depuis longtemps vilipendé par les vecteurs de la propagande patriotique. Depuis la guerre franco-prussienne, de nombreuses générations ont ainsi été nourries par un esprit de revanche qui finalement justifie les élans patriotiques et la mobilisation de l'ensemble des composantes de la Nation. Cette enculturation ${ }^{6}$ a été d'autant plus efficace à La Réunion que le conditionnement repose sur deux prédicats normatifs fortement ancrés dans les esprits : la revendication d'une appartenance nationale et le sens du devoir hérité des ancêtres.

Les responsables politiques de l'île s'empressent donc de rappeler la nécessité d'un total dévouement à la Patrie, quitte à laisser de côté les traditionnelles querelles partisanes pour faire front face à l'ennemi. C'est ainsi que le Comité fédéral du Parti radical et radical-socialiste informe par une motion patriotique en date du 11 août 1914 que la guerre qui vient d'être déclarée nécessite une collaboration active de la part des mobilisés mais également de la part des civils qui doivent faire preuve d'une certaine humanité :

Le Comité Fédéral du Parti radical et radical-socialiste de l'île de La Réunion, considérant que sans déclaration de guerre, l'Allemagne a commencé les

5. Rachel Mnémosyne, "La Réunion et les Réunionnais dans la Grande Guerre ", in Yvan Combeau (dir.), La Réunion sous la Troisième République. Une colonie républicaine (1870-1940), SaintAndré, Océan éditions, 2005, p. 102.

6. Processus par lequel l'individu assimile durant toute sa vie les traditions de son groupe et agit en fonction de ces traditions. 
hostilités contre la France, adresse à la mère Patrie et à son représentant dans la colonie, au nom de la population républicaine, l'expression de son entier dévouement et des vœux ardents qu'il forme pour le succès des armes françaises, c'est-à-dire du droit et de la liberté ; adresse également avec émotion, aux représentants des Nations amies, belligérants et neutres, l'expression de sa gratitude ; félicite la population de la colonie d'avoir répondu avec enthousiasme à l'ordre de mobilisation; demande à tous les citoyens de faire trêve de leurs dissensions politiques pendant la durée de la guerre, fait appel au patriotisme et à l'humanité des commerçants pour épargner un surcroît de souffrances à la population par une hausse injustifiée et arbitraire du prix des denrées alimentaires ${ }^{7}$.

De même, par un télégramme adressé au gouverneur, le maire de Saint-Denis ${ }^{8}$ déclare au nom de son conseil municipal et de l'ensemble de ses administrés que "les cœurs battent à l'unisson ", qu'ils sont " remplis d'une joie profonde et patriotique $»^{9}$. Il tient également à exprimer ses sentiments d'admiration aux familles de ceux qui sont partis pour défendre la mère Patrie et conclut sa déclaration par un vibrant hommage aux valeurs défendues par la République. Il s'agit donc à la fois de défendre la Patrie et l' "idéal de liberté, de justice et de droit $^{10}$ " que professe la République. Le Conseil général rédige également une motion que le gouverneur transmet au ministre des Colonies pour rappeler l'« attachement filial de [la] Colonie à [la] France, [le] patriotisme intégral [de] ses habitants ${ }^{11}$ » et le fait que l'île doit donner "sa part contributive en soldats qui puissent les élever au rang des plus dignes parmi les Français des colonies ${ }^{12}{ }^{\prime}$. Cette demande s'inscrit dans la volonté des Réunionnais de "rester les égaux de leurs frères de la métropole et de défendre comme eux la Patrie menacée ${ }^{13} »$. Les lettres de soutien sont le plus souvent adressées au gouverneur en qualité de représentant de l'État et seul capable de transmettre les vœux des Réunionnais aux plus hautes instances politiques. Dans une lettre de soutien en date du 8 aout 1914, un groupe de citoyens, dont on peine à reconnaître les signatures, s'inscrit également dans cette veine :

La France vient d'être l'objet d'une agression brutale. L'empereur allemand lance ses armées sur elle et sur des pays voisins et amis. Ces actes odieux, commis en violation de traités solennels, sont sans précédents dans l'histoire du monde. Dans les circonstances douloureuses que traverse la Patrie, nous tenons, Monsieur le gouverneur, à vous dire que nos cœurs battent à l'unisson de ceux de nos frères de France.

7. Motion patriotique du Parti radical et radical-socialiste de l'île de La Réunion, 11 août 1914, Archives Départementales de La Réunion (ADR) R 50.

8. Chef-lieu de la colonie.

9. Télégramme du maire de Saint-Denis au gouverneur du 12 novembre 1918, ADR, R136.

10. Ibid.

11. Lettre du Conseil général au ministre des colonies du 22 août 1914, Archives nationales de l'outre-mer (ANOM) FM/SG C528D6183.

12. Ibid.

13. Ibid. 
Nous vous prions de faire connaitre au gouvernement de la République et à son éminent président, M. Poincaré, que nous avons une confiance absolue dans leur patriotisme éclairé. Vous voudrez bien encore leur transmettre les vœux ardents, que nous formons pour le succès de la France, qui, comme le dit le message présidentiel, représente encore une fois aujourd'hui, devant l'univers, la Liberté, la Justice et la Raison ${ }^{14}$.

Le témoignage d'Adrien Jacob de Cordemoy permet de rendre compte de la ferveur déclenchée par l'annonce de la mobilisation dans le chef-lieu dionysien :

C'était le dimanche soir [...] au théâtre de Saint-Denis, on donnait La Petite Mariée $^{15}$. Les musiciens au nombre desquels je me trouvais, échangeaient leurs impressions. Dans l'air flottait ce je ne sais quoi qui précède les grands événements. Les poitrines étaient oppressées et chacun semblait chercher dans le regard de son voisin la solution du problème de l'avenir. Une même pensée absorbait la salle tout entière : la guerre éclaterait-elle ?

Tout à coup, dans la rue, près de la Gendarmerie, un clairon sonne la générale. On croit tout d'abord à un incendie, car il était coutume, à cette époque, d'annoncer le feu par une sonnerie semblable. Mais l'on apprend aussitôt, hélas, que c'était la mobilisation, et le clairon parcourt la ville accompagnée d'une jeunesse enthousiaste. [...]

Après les premiers moments de consternation, les artistes reprennent leurs esprits. Que vont-ils faire ? Et aussitôt, il est convenu que le spectacle aurait lieu, mais qu'avant de lever le rideau, on chanterait la Marseillaise. Les spectateurs debout, écoutent dans un profond recueillement l'hymne national, puis manifestent à haute voix leurs sentiments patriotiques. À la fin du spectacle, la troupe, alors au grand complet, car les artistes qui ne jouaient pas étaient accourus, entonne à nouveau le chant de Rouget de Lisle. Des tonnerres d'applaudissements répondent à ce geste ${ }^{16}$.

Grâce aux souvenirs de Charles Foucque, nous pouvons également esquisser quelques traits de l'effervescence populaire et des réactions provoquées par l'annonce de la guerre à Hell-Bourg dans le cirque de Salazie. Son témoignage est d'autant plus précieux que les sources restent lacunaires pour les populations isolées de l'île :

Mais voici que sur la terrasse du vieux bureau des postes, dans la grand'rue, des gens s'interpellent, s'agitent, discourent. Qu'est-ce donc? Le câble vient d'apporter la terrifiante nouvelle : c'est la guerre ! Que le village changea bientôt d'aspect! Ainsi que dans un centre de dépression les nuages se précipitent violents et bruyants, les sentiments les plus chauds, les plus troublantes images envahirent les cœurs et les esprits. L'exubérance de la race éclata, la petite paroisse entra en frénésie. C'est à voir ces "moreng ${ }^{17}$ " contre les ennemis

14. Lettre de soutien au gouverneur, ADR R 136.

15. Opéra-bouffe en 3 actes d'Eugène Leterrier et Albert Vanloo sur une musique de Charles Lecocq de 1875.

16. Jacob de Cordemoy Adrien, "L'île de la Réunion et la Grande Guerre ", Bulletin de l'académie de la Réunion, 1939, vol. 16, pp. 27-62.

17. Danse guerrière d'origine malgache. 
imaginaires, coups de pieds par-ci, coups de tête par-là, poings crispés et talons levés - à entendre ces menaces et ces insultes en un langage imagé et cocasse. À l'écart, de rares avertis et leurs mères soucieuses supputaient discrètement les malheurs qui allaient accabler le pauvre monde. Pour eux le globe serait en feu ; deux civilisations contraires étaient aux prises, les peuples se rangeraient sous l'un ou sous l'autre étendard selon leurs affinités et leur culture ; tout serait bouleversé. Beaucoup demeuraient sombres. Leurs pensées, pardelà les mers, étaient auprès des enfants sous les drapeaux, aux zouaves, aux marsouins. Car de nombreuses familles, chaque année, envoyaient leurs fils faire le service militaire en France et y terminer leurs études ${ }^{18}$.

Terreur, forfanterie, trouble, frénésie et inquiétude témoignent des sentiments les plus chauds dans cette petite commune rurale qui se démarque par bien des traits de la solennité relatée par Adrien Jacob de Cordemoy. Dans les écarts de l'île, la nouvelle a cependant mobilisé l'ensemble des populations qui, si l'on en croit le témoignage de Marie-France Dijoux, ont été informées par les cloches des églises et pour les lieux les plus reculés par les crécelles que les enfants faisaient tourner ${ }^{19}$. Cette mobilisation donne le ton quant aux sentiments éprouvés par les Réunionnais durant la guerre.

Cette question a été très largement débattue et sujette à des analyses exhaustives. Les travaux de Prosper Ève ${ }^{20}$ et ceux de Rachel Mnémosyne ${ }^{21}$ ont mis en évidence la ferveur patriotique dans laquelle ont vécu les Réunionnais durant le conflit. À quelques nuances près, cette ferveur a été partagée et ne s'est jamais éteinte si l'on en croit leurs conclusions. Si l'expérience combattante peut nous permettre de nous inscrire dans une vision patriotique belliqueuse partagée avec les mobilisés européens, il semble plus intéressant de nous concentrer sur une vision de l'arrière et d'aborder les psychoses provoquées par la peur des ennemis. Par ce biais, nous entendons mettre en évidence la prégnance du phénomène guerrier malgré l'absence de combats et la nécessité pour les Réunionnais de se protéger de supposés ennemis intérieurs, afin de rendre tangible leur participation patriotique au conflit.

\section{LA PARTICIPATION À L'EFFORT DE GUERRE}

La peur de l'ennemi se reflète avant tout dans la psychose d'une attaque de l'île par des croiseurs allemands. Bien qu'éloignée du cœur des opérations militaires, la rumeur s'amplifie chaque jour autour de fausses informations ou d'erreurs journalistiques qui laissent entrevoir l'ampleur des

18. Charles Foucque, Quelques notes et souvenirs : 1914-1918, Saint-Denis, Impr. Drouhet, 1930, p. 4.

19. Cf. Sulliman Issop, "D'hier et d'aujourd'hui" de Marie-France Dijoux, GRAHTER Ed., 2004.

20. Prosper Ève, La Première Guerre mondiale vue par les poilus réunionnais, Saint-Denis, Éditions CNH, 1992

21. Rachel Mnémosyne, Les Soldats réunionnais dans la Grande Guerre, 1914-1918, thèse ss.-dir. Yvan Combeau, Université de La Réunion, 2006. 
hantises et la force des préjugés. Malgré l'éloignement de l'île du cœur des opérations militaires, la psychose de guerre s'installe, défiant ainsi les lois de la géographie. Cette agitation est directement alimentée par les autorités centrales, comme le suggère cette circulaire de Gaston Doumergue du 25 septembre 1914 au sujet des centres d'information d'outre-mer :

Le ministre de la Marine vient de me faire connaitre que le Gouvernement anglais serait désireux que nos gouverneurs $[\ldots]$ centralisent tous les renseignements maritimes et les communiquent aux consuls britanniques [...]. Ce service de renseignement parait d'autant plus s'imposer que le Gouvernement de la Grande-Bretagne a établi des services de cette nature dans la plupart de ses colonies ${ }^{22}$.

La circulaire précise qu'il s'agit des pavillons allemands et autrichiens et que ces précautions ne constituent que le reflet d'une politique sécuritaire logique en temps de guerre. Malgré l'absence de danger immédiat, les relais de l'administration coloniale établissent pourtant des enquêtes dans cette optique, accentuant de fait le sentiment d'insécurité. Le capitaine Deroche, commandant du détachement de Gendarmerie de La Réunion envoie ainsi un rapport, classé secret, au gouverneur de l'île concernant une possible attaque des Allemands sur l'île dès le 13 août 1914 :

J'ai l'honneur de vous soumettre les dispositions qui pourraient être prises éventuellement en cas de l'arrivée d'un bâtiment allemand sur les côtes de l'île de la Réunion :

L'ennemi n'observe pas les conventions internationales et bombarde un point de l'île, puis tente un débarquement. Solution : défense à main armée.

L'ennemi respectueux des conventions internationales réquisitionne sans acte d'hostilité préliminaire, ou débarque provisoirement. Solution : selon toute probabilité, les régions intéressées seront comprises dans la zone Saint-Denis, le Port, Saint-Paul. Partant, les notables de ces localités décideront, en conseil sous la direction de la haute administration, quelle sera la conduite à tenir pour sauvegarder les intérêts généraux de l'île.

$\mathrm{Au}$ point de vue militaire, seul le premier cas est à envisager à l'heure actuelle $^{23}$.

En dépit de toute logique stratégique, seule l'invasion est perçue comme valide par les autorités. Une force de cent cinquante hommes ${ }^{24}$ est ainsi mobilisable pour faire face à une attaque des positions stratégiques de l'île même si l'armement et les munitions semblent très largement insuffisants ${ }^{25}$ pour pouvoir les équiper. En cas de bombardement, on compte évacuer les populations sur les " écarts » et répartir les vivres sur plusieurs points de

22. Correspondance du gouverneur, ADR 1 M1683.

23. Dispositions en vue de l'arrivée éventuelle d'un navire allemand à la Réunion, 1914, ADR R 136

24. 60 hommes de la gendarmerie, 15 hommes de l'infanterie coloniale, 10 hommes des douanes, 10 hommes des gardes forestiers et 55 mobilisés de l'armée territoriale qui sont affectés à des tâches d'administration militaire locale.

25. Le commandant Deroche ne comptabilise que cent-cinquante fusils dont certains sont en service depuis plus de quarante ans! 
l'île. Pour prévenir un quelconque débarquement, le capitaine Deroche préconise de positionner des vigies à Saint-Denis, au Port, à Saint-Leu et à Sainte-Rose mais également d'obstruer l'entrée du port par un câble amarré aux blocs de la jetée. Les autorités de l'île s'enferment de la sorte dans une forme de paranoïa où les plus grands périls sont envisagés. Certes, il s'agit de répondre aux injonctions émanant des autorités centrales, cependant sur une île où la peur est quasiment consubstantielle à l'identité réunionnaise ${ }^{26}$, il n'en faut guère plus pour alimenter les rumeurs d'une invasion. La presse s'empare du sujet et accentue la psychose générale. C'est ainsi que les journaux parlent désormais de piraterie allemande, renvoyant à une mémoire collective douloureuse autour de l'insécurité des mers. La flotte allemande déployée se résume pourtant à sept navires dans le Pacifique, à cinq dans l'Atlantique et seulement deux dans l'océan Indien. La force de frappe est cependant bien réelle puisque cette flottille a coulé ou capturé 240000 tonneaux jusqu'à la fin de l'année 1914.

Concernant l'océan Indien et les inquiétudes des Réunionnais, le Koenisberg et l'Emden vont ainsi défrayer la chronique durant les trois premiers mois du conflit. Le directeur de la Banque de La Réunion est tellement affolé qu'il suggère un repli de l'or de la colonie sur l'île Maurice. Le gouverneur, qui en réfere immédiatement au ministre des Colonies, lance un appel au calme et suggère de cacher l'or dans le cirque de Salazie. Faisant face à des informations contradictoires, la presse s'inquiète du moindre retard pris par un paquebot ${ }^{27}$ en route pour La Réunion ${ }^{28}$. Lorsque le Koenisberg coule le croiseur Pegasus en rade de Zanzibar, la presse ${ }^{29}$ en fait ses gros titres et développe la thèse d'une insécurité des lignes maritimes occidentales de l'océan Indien :

C'est un véritable croiseur fantôme que ce Königsberg; et la série de ses exploits commence à s'allonger beaucoup. Il est fort capable un de ces jours, de tourner son regard de notre côté et de venir se ravitailler chez nous ${ }^{30}$.

Le soulagement ne viendra qu'en février 1915 lorsque ce dernier sera coulé dans une rade de Madagascar. Cet affolement est réactivé par les prouesses de l'Emden dont on conte les exploits à la " une " des journaux. Il a ainsi coulé huit navires en septembre 1914, puis six le mois suivant. La peur devient une réalité pour les Réunionnais lorsque l'on apprend le bombardement de

26. Cf. Prosper Ève, Île à peur. La peur redoutée ou récupérée à La Réunion des origines à nos jours, Saint-André, Océan édition, 1992.

27. Dans ses souvenirs, Charles Foucque évoque cette psychose lorsqu'il prend un paquebot pour se rendre à Madagascar : " Et ce fut la haute mer entre le ciel et l'eau, le silence éternel des espaces infinis, tandis que l'île se perdait dans la flambante transparence de midi. La nouvelle que le croiseur allemand Koenigsberg était dans les parages circule bien vite à bord, exacerbant encore les imaginations. Mais on scrute vainement l'horizon, le vaisseau de guerre reste fantôme ", in Quelques notes et souvenirs : 1914-1918, op. cit., p. 160.

28. Inquiétude qui concerne notamment le Djemmah en août 1914, voir Nouveau journal de l'île de la Réunion du 19 août 1914, ADR, 1PER52/10.

29. Nouveau journal de l'île de la Réunion, 24 septembre 1914, ADR, 1PER52/10.

30. L'Action de l'île de la Réunion, 19 septembre 1914, ADR 1PER54/7. 
Madras en septembre ${ }^{31}$. La fin du cauchemar ne sera effective qu'en novembre après le torpillage de l'Emden au Sud-Ouest de Java.

Face à ce flot d'informations, la population se persuade donc de l'arrivée imminente de cette flottille allemande sur les rivages réunionnais. C'est ainsi que le 22 septembre 1914, un navire de pavillon étranger se présente devant le port. La panique est perceptible sur les quais et une foule nombreuse vient aux nouvelles. Le responsable du port envoie des messages par signaux lumineux afin de connaître l'identité du navire, et l'on assiste alors à une incompréhension et un mouvement de foule avant de comprendre qu'il s'agit d'un navire anglais en partance pour Maurice ${ }^{32}$ ! De même, en novembre 1914, les Saint-Paulois croient entendre les coups de canon du Königsberg durant une nuit de tempête, confondant ainsi le ressac bruyant provoqué par les conditions climatiques avec un bombardement de l'île ! Certes, les journalistes ont bien conscience de l'exagération de la peur mais :

Sans être impressionnables à l'excès, nous avançons, en ce qui nous concerne, que ce n'est pas sans un frisson à fleur de peau, que nous songeons à la visite, peu improbable en somme, puisque leurs croiseurs continuent à évoluer dans la mer des Indes, des brutes allemandes qui ne se contenteront peut-être pas de beaucoup d'or, et en voudront peut-être aux vies humaines, qui ne comptent pas pour [eux], quand ils débarqueront en maîtres dans une île sans défense ${ }^{33}$.

Pour rassurer la population, devant ces brutes pour qui les vies humaines ne comptent pas, les journaux proposent d'établir un bilan des forces navales en présence durant le conflit :

Maintenant que l'état de guerre entre l'Angleterre et l'Allemagne est officiellement proclamé, nous nous devons de dire à nos lecteurs que rien ne parait menacer notre île. Les bâtiments allemands sont en infériorité numérique manifeste vis-à-vis des flottes anglaises et russes combinées, et la fantaisie dût-elle leur venir de quitter la mer du Nord pour tenter un raid dans l'océan Indien, ils trouveraient leur chemin barré. De même, les Autrichiens et éventuellement les Italiens auront assez de mal à assurer leur propre sécurité pour ne pas songer à nous. Quant au vieux croiseur allemand qui se trouve dans l'océan Indien, on peut être sûr qu'il ne mettra pas le cap sur l'île Maurice, la sachant défendue par des croiseurs anglais qui le mettraient en pièce ${ }^{34}$.

Malgré ces explications rassurantes, les autorités de l'île décident cependant de parer à toute éventualité en arrêtant les dispositions suivantes :

1. Si navire ennemi manifestait intention pénétrer dans le port, la plus forte drague du Chemin de Fer et Port de La Réunion serait coulée dans la passe afin de l'empêcher si possible de ravitailler en charbon à la Pointe des Galets où se trouvent les dépôts de combustibles.

2. Les fonds du trésor et de la Banque de La Réunion vont être transportés incessamment dans les hauteurs de Salazie où un caveau a été aménagé à cet effet.

31. Nouveau journal de l'île de la Réunion, 27 septembre 1914, ADR, 1PER52/10.

32. Nouveau journal de l'île de la Réunion, 23 septembre 1914, ADR, 1PER52/10.

33. La Patrie créole, 10 novembre 1914, ADR 1PER45/27.

34. Nouveau journal de l'île de la Réunion, 10 août 1914, ADR 1PER 52/10. 
3. Aussitôt navire ennemi reconnu, je câblerai à Tananarive - Maurice Durban - Zanzibar - Mombassa pour que soient prévenus navires de guerre français ou anglais opérant dans ces régions ${ }^{35}$.

Cette psychose va durer tout le long du conflit et entretenir le mythe d'une possible attaque. C'est ainsi qu'en 1916, le gouverneur de Madagascar envoie au gouverneur de La Réunion, un étrange message codé, sorte d'esperanto surprenant où il indique :

93. Capitaine Vapeur Anglais Brigitta qui décharge charbon créolo collevisti sosaquemos et qui doit revenir Lorenço Marquez aurait reçu anticorro amymonius assuringly cubile assumpting sfumatezza alloppiano assopito anxietudo Mozambique crois devoir vous scoperta concliclam en vous priant me la arillado apesarando Duprat ${ }^{36}$.

Immédiatement, le gouverneur de La Réunion prend des mesures et confie l'enquête au commandant de la brigade du Port :

Une lettre du maréchal des logis Prignac, commandant de la brigade du Port, est adressée au gouverneur de La Réunion pour lui signaler qu'un bateau de guerre allemand rode aux alentours, information qu'il détient du navire anglais Le Brigitta: "Ce navire allemand, en raison des transports de troupes qui s'effectuent de Madagascar pour la France et qui passe par le Cap pourrait fort bien attendre ces transports s'ils n'étaient prévenus. " [...] Cette communication de la part du Capitaine anglais m'a été faite confidentiellement ${ }^{37}$.

Le 5 février 1917, suite à un câblogramme reçu du gouverneur de Madagascar, le gouverneur Duprat demande au Colonel Lamiable de prendre des dispositions « en vue [...] approche corsaire et débarquement Garbit $^{38}$ ». La peur que les Réunionnais ne soient victimes de ces torpillages trouve finalement son triste épilogue en mai 1917, lorsque le Yarra, qui transportait à son bord six cents Réunionnais pour l'Europe, a été victime de cette guerre maritime en Méditerranée.

Les éléments déclencheurs de cette psychose sont donc à rechercher avant tout dans le manque d'informations et le traitement de celles-ci. S'il est évident que la rupture du câble reliant La Réunion à Madagascar au cours du mois d'août $1914^{39}$ a provoqué un réel retard dans la diffusion des faits de guerre, les journalistes

se demande[nt] si, en outre de la censure rigoureuse exercée en France et qui ne permet la transmission télégraphique que des faits dont on veut bien que nous soyons informés, il n'en existe pas une autre, sur place, qui a pour effet

35. Dépêche télégraphique du 11 novembre 1914, ANOM FM/SG c528d6183.

36. Ce courrier du 8 juillet 1916 (ADR, R 50) évoque l'attaque d'un vapeur britannique dans le canal du Mozambique. L'utilisation de termes français, anglais, espagnols, italiens et latins devait rendre obscure toute lecture.

37. Lettre du maréchal des logis Prignac, commandant de la brigade du Port au gouverneur de La Réunion, le 7 juillet 1916, ADR R50.

38. Lettre du gouverneur au colonel Lamiable, 5 février 1917, ADR R 136.

39. Nouveau journal de l'île de la Réunion, 21 août 1914, ADR, 1PER52/10. 
de rendre un peu moins intelligibles encore les rares nouvelles qui filtrent si péniblement ${ }^{40}$.

En effet, le contrôle de la presse est une réalité depuis qu'un décret de censure a été voté le 5 août 1914, cependant l'arrêté n'a été pris pour La Réunion que le 3 octobre et publié, dans le Journal Officiel de l'île, le 9 du même mois. Une certaine liberté est donc possible jusqu'à cette date et ne justifie en rien les inquiétudes énoncées. La censure est entre les mains du gouverneur et orchestrée par le commandant de la région militaire. Les textes stipulent l'interdiction de publier des renseignements de nature à nuire aux relations avec les pays alliés, neutres, ou relatifs aux négociations politiques, d'attaquer les officiers, de reproduire des articles parus dans la presse étrangère, de divulguer les lieux de décès des hommes sur le front, de mentionner l'expérience combattante, de publier des interviews de généraux et suggère enfin de surveiller tout ce qui pourrait correspondre à de la propagande pour la paix.

La censure explique sûrement en partie les nombreuses phobies engendrées par le manque d'informations. Comme la population de l'île est dans sa majorité illettrée, les informations circulent de bouche à oreille et sont relayées à travers un filtre imaginatif plutôt prolixe. Nous pouvons émettre l'hypothèse que ces agitations ont essentiellement pour but de mobiliser l'opinion qui en retour ne peut qu'accepter les mesures coercitives mises en place par les autorités. Dans le même temps, nous pouvons imaginer qu'il s'agit de montrer à la métropole que les Réunionnais ne sont pas épargnés par les souffrances de la guerre et communient de la sorte avec le reste de la Nation.

\section{LES FIÈVRES OBSIDIONALES}

Si le principe d'identification nationale se nourrit d'un besoin d'appartenance et de reconnaissance, il peut également prendre sa source dans l'exclusion et l'inimitié envers les ennemis de la Nation qu'ils soient réels ou imaginaires. Ces sentiments peuvent déboucher sur des fièvres obsidionales, c'est-à-dire des psychoses collectives ressenties par une population qui se sent assiégée et qui, pour notre cas, cherche des ennemis pour légitimer son combat patriotique malgré l'éloignement de la guerre. En effet, conformément aux principes de l'histoire culturelle qui s'attache à étudier "l'ensemble des représentations collectives propres à une sociétét ${ }^{41}$ ", nous pouvons nous interroger sur la place de la guerre dans les processus de construction identitaire et sur les comportements des acteurs face à la montée en puissance de la violence. La notion de déviance prend ici tout son sens : elle définit des attitudes non conformes par rapport à des normes

40. Nouveau journal de l'île de la Réunion, 27 août 1914, ADR, 1PER52/10.

41. Cf. Pascal Ory, L'Histoire culturelle, Paris, Puf, coll. "Que sais-je ? ", 2007. 
définies et s'inscrit dans un comportement de transgression qui aboutit pour notre sujet à une modification des sociétés en temps de guerre. La Réunion n'a pas échappé à ce processus, intégrant de nouvelles valeurs censées prouver le consensus patriotique et déterminer une réelle volonté de participer à l'effort de guerre.

Éloignée des opérations militaires, la population de l'île adopte en effet de nouveaux comportements et développe des suspicions qui tendent à définir un ennemi à la fois extérieur mais aussi intérieur. La peur d'être envahi, le délire psychotique autour de l'espionite, la stigmatisation des ennemis de la Nation vont ainsi devenir des révélateurs d'une nouvelle conscience patriotique.

L'analyse des ressentiments populaires permet d'entrer pleinement dans le domaine de l'imaginaire et des représentations mentales. Il s'agit donc de pointer l'ensemble des actes rendant sensibles l'appartenance à une idéologie, à un modèle type de pensée troublé par les idées préconçues et les différences de culture. Psychose et espionite s'inscrivent ainsi dans un contexte de craintes réciproques entre les différents groupes ethniques qui cherchent leurs marques durant cette période de tensions et de suspicions. Au-delà d'un héritage, il s'agit certainement d'une volonté d'en " découdre " avec un ennemi irréel qu'il convient de rendre tangible afin de participer à l'effort de la communauté nationale.

Pendant le conflit, l'ennemi intérieur représente une figure majeure de la culture de guerre. Parce que celle-ci exige la mobilisation de la nation tout entière, l'arrière représente l'une des sources de la force militaire, de sorte que la peur d'une quelconque déstabilisation entraîne des réactions violentes. Comme le souligne Gundula Bavendamm :

L'accusation d'affaiblir la volonté de défense nationale par la subversion, la terreur, le sabotage, l'espionnage ou la trahison vise plus particulièrement les minorités nationales et les groupements politiques et idéologiques marginaux. Dans les sociétés en guerre, il n'y a qu'un tout petit pas à franchir entre le nonconformisme et le soupçon de collaboration avec l'ennemi ${ }^{42}$.

On retrouve bien évidemment cette suspicion dans les autres États belligérants. Pensons à l'Alien Restriction Act britannique, voté le 5 août 1914, qui a permis notamment l'internement des Allemands et des Autrichiens, mesure qui est d'ailleurs en vigueur dans la colonie mauricienne.

Nous retrouvons cette propagande sur l'île de manière réduite à l'encontre de la communauté z'arabe $e^{43}$. Ceci explique les mesures de contrôle des courriers et la surveillance des périodiques venant de l'étranger. Les

42. Gundula Bavendamm, "L'ennemi chez soi ", p. 751, in Audoin-Rouzeau Stéphane, Becker Jean-Jacques (dir.), Encyclopédie de la Grande Guerre 1914-1918, Paris, Bayard, 2004.

43. Pierre-Éric Fageol, "Les Boches coloniaux". Les commerçants asiatiques sous la vindicte populaire à La Réunion pendant la Grande Guerre ", Guerres mondiales et conflits contemporains n 243 , 2011, pp. 5-34. L'appellation "z'arabe » renvoie à la communauté musulmane originaire du souscontinent indien et plus spécifiquement du Gujarat. De confession sunnite, les Z'arabes ont migré à La Réunion à partir du milieu du XIX ${ }^{\mathrm{e}}$ siècle et se sont distingués progressivement par rapport aux autres composantes indiennes de l'île de confession Hindou que l'on dénomme Malbars. 
télégrammes privés doivent être écrits "seulement en langage clair. Ils doivent offrir par eux-mêmes un sens intelligible pour le service télégraphique. Ils sont soumis à la censure et sujets à retard ${ }^{44}$ ». Un télégramme du 10 août 1914, provenant du ministère des colonies et adressé au gouverneur, stipule ainsi que tout document utilisant un langage secret ou rédigé dans une langue étrangère est interdit ${ }^{45}$. C'est pourquoi, la gendarmerie est alertée de la réception d'un journal en ourdou par un certain Issop Casim dans la commune de Saint-Denis. Ne pouvant être traduit, le journal est censuré et les expéditions sont stoppées rapidement. Provenant des Indes britanniques, répondant au titre évocateur de "Trahison " ou "Tromperie ", ce journal reste tout d'abord une énigme pour les autorités de l'île. Les suspicions sont d'autant plus importantes que le commerçant qui le reçoit est bien établi dans le commerce sur Saint-Denis et qu'il fait partie des cinq initiateurs de la fondation de la mosquée dionysienne ${ }^{46}$. La maitrise de l'ourdou, langue liturgique peu connue des fidèles de La Réunion, suppose que ce commerçant soit doctement instruit en théologie islamique. La coutume voulant que les imams de cette période soient issus de la diaspora indienne, on peut supposer qu'Issop Casim a conservé des contacts avec le sous-continent indien.

Fig. 1 : Journal censuré dès le début du conflit, A.D.R., R 50.

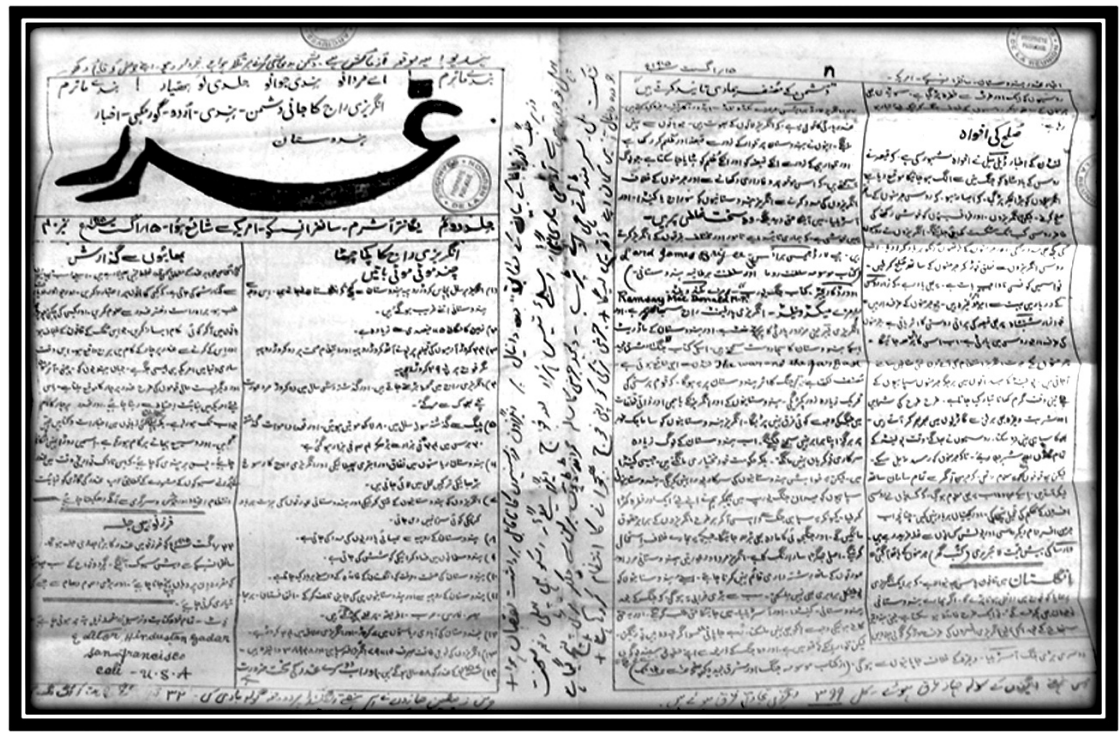

44. Nouveau Journal de l'île de la Réunion, 7 août 1914, ADR 1PER52/10.

45. Télégramme du 10 août 1914 du ministère des Colonies au gouverneur, ADR, R 50

46. Marie-France Mourrégot, L'Islam à l'île de La Réunion, Paris, L'Harmattan, 2010, p. 365. 
Le sous-titre interpelle l'ensemble des communautés indiennes et demande de se soulever et de refouler l'envahisseur : "Ô les hommes, jeunes Indiens, prenez vite vos armes ${ }^{47}$. "

Les articles suivants suggèrent un livre noir de la colonisation britannique dans lequel sont sanctionnés l'absence de politique de développement (la pauvreté augmente) et le manque d'efficacité sanitaire (" durant les seize dernières années huit millions de victimes d'épidémies »). L'accusation porte également sur la politique ethnique menée par les Britanniques qui vise à soulever les hindous contre les musulmans. Cette thématique a déjà été étudiée par ailleurs ${ }^{48}$ et invite les puissances de la Triple Entente à faire preuve de vigilance car

faute de pouvoir ou de vouloir intervenir directement, les agents des puissances centrales tentent, au moins, d'ébranler les esprits des populations. Des tracts invitent les musulmans à se soulever contre les puissances coloniales, et à soutenir l'effort de guerre de l'Allemagne et de son allié ottoman. Ils soulignent les défaites ou les échecs des Alliés, qu'ils opposent aux victoires de l'Allemagne, dont la puissance est largement magnifiée. Rédigés dans les langues des pays auxquels ils sont destinés, ils émanent d'officines à la tête desquelles sont souvent placés des exilés en Allemagne ou en Suisse, mais à la rédaction desquels collaborent aussi des universitaires orientalistes allemands ${ }^{49}$.

C'est pourquoi, les autorités françaises tentent de recouper leurs informations avec celles de leurs alliés afin de rendre plus efficace leurs institutions de renseignement. Pour La Réunion, une circulaire de Gaston Doumergue enjoint ainsi le gouverneur de La Réunion à partager ses informations avec les autorités consulaires sur l'île.

La hantise de l'espionnage et de la subversion incite les autorités réunionnaises à rechercher l'action occulte d'agitateurs ennemis. Les Allemands et les Italiens sont évidemment les premiers suspects. Même s'ils sont installés depuis longtemps sur l'île, qu'ils soient diplomates ou négociants, ils subissent les foudres de l'administration qui les accuse de fomenter des actions subversives. Ce climat domine surtout au début du conflit et provoque une peur parfois hystérique de l'espion qui s'accompagne d'une vague de dénonciations en tout genre frisant parfois le ridicule. Une réclame pour une maison allemande, envoyée de Paris au président de la chambre de commerce de La Réunion met ainsi en émoi les autorité $s^{50}$. Les animosités à l'encontre des germanophiles impliquent également un contrôle des habitants d'origine allemande sur l'île. L'exemple est d'abord donné par le gouvernement de Madagascar et la presse réunionnaise enjoint les autorités à en faire de même :

Le gouverneur général de Madagascar a pris de sévères mesures contre les Allemands résidant sur les différents points du territoire de la colonie. Comme

47. Traduction de l'ourdou établie par l'imam de Saint-Paul Shokatali Limbada en mai 2011.

48. Cf. Marie Rousseau, "La propagande panislamique pendant la Première Guerre mondiale ", Mémoire de maîtrise, université Paris-IV, 2004.

49. Jacques Frémeaux, Les Colonies dans la Grande Guerre. Combats et épreuves des peuples d'outremer, Paris, Soteca, coll. «14-18 Éditions », 2006, p. 270.

50. ANOM, FM/SG/REUc528 d6216. 
de dangereux pestiférés les sujets allemands venant de l'intérieur de l'île et de la côte Est, ont été internés à l'îlot Prune, sous la garde d'un peloton de tirailleurs malgaches ${ }^{51}$.

Charles Foucque relate ces mesures discriminatoires prises sur la Grande Île non sans faire remarquer que, finalement, elles rendent justice aux Malgaches puisque les Allemands avaient accaparé la plus grande partie de la colonie. Pour légitimer ces mesures, les "boumaines " - "billes rouges "- sont accusés d'espionnage car, comme le souligne de nouveau Charles Foucque :

N'avait-on pas découvert dans leurs effets séquestrés " des costumes d'officiers et même de gouverneur ", et retrouvé, cachés dans leurs archives, des plans et des rapports secrets sur les êtres et les choses de ce Madagascar qu'ils convoitaient maintenant? Les jeunes ne voulaient plus, comme leurs aînés, se contenter d'établissements de commerce; ils étaient de la nouvelle école allemande de domination complète. Leur pays entendait ne plus dépendre de personne, être chez soi partout. Aux colonies tout étant pris, il se trouvait conduit à jeter les yeux sur les possessions d'autrui, surtout celles des Nations qui comme la France, selon sa prétentieuse affirmation, étaient incapables ou détenaient des terres sans en tirer parti, comme eût fait une race digne de cette fortune, telle que la race allemande ${ }^{52}$.

À La Réunion les suspicions se portent essentiellement sur un Allemand et deux Italiens. Le 12 août 1914, le capitaine Deroche est ainsi mandaté pour mener une enquête sur ces trois ressortissants étrangers. La pression exercée par l'opinion publique n'est pas étrangère à ce zèle, et le journal Le Vieux Tambour n'hésite pas à entretenir la psychose :

Et dire que dans notre pays il y a des Prussiens... Hélas! Oui, dans ce cher petit pays, île ensoleillée dont l'éternel printemps nous donne l'illusion de l'éternelle jeunesse, il y a des créoles à tête de Prussien, à gueule de Uhlans. Ils disent ces mauvais créoles que la France devrait recevoir une tripotée, au moins disent-ils, nous serions débarrassés de cette sacrée République ${ }^{53}$.

La suspicion touche ainsi de manière grotesque et parfois paranoïaque un certain Rohnstadt comme l'illustre ce courrier du gouverneur Cor au ministre des colonies :

J'ai l'honneur de vous transmettre ci-joint, à toutes fins utiles, une lettre qui m'a été adressée par le Sieur Rohnstadt, sujet allemand, actuellement interné à l'hôpital colonial de Saint-Denis. Cet individu demande à bénéficier d'un permis de séjour ; il invoque à l'appui de sa requête son état de santé précaire, ainsi que son mariage avec une française. Je crois utile de vous rappeler, Monsieur le ministre, qu'à la date du 31 décembre, je vous ai signalé que Rohnstadt était âgé de plus de soixante ans, et en outre impotent; en l'internant je me suis

51. La Patrie créole, 16 septembre 1914, ADR 1PER45/27. p. 14.

52. Charles Foucque, Quelques notes et souvenirs : 1914-1918, Saint-Denis, Impr. Drouhet, 1930,

53. Le Vieux Tambour, 3 septembre 1914, ADR, 1PER56. 
borné à exécuter les ordres que vous m'avez donnés dans vos câblogrammes des 4 janvier et 24 février 1915. H. Cor, 20 juillet $1915^{54}$.

Les suspicions établies contre Frédéric Rohnstadt mettent en alerte les services du ministère des Colonies qui informent le député réunionnais Lucien Gasparin des résultats de l'enquête menée à son encontre :

J'ai l'honneur de vous faire connaître que d'après des renseignements très précis qui ont été fournis au Département par l'administration locale, le sieur Rohnstadt, quoique né en Russie et marié à une française, n’en est pas moins allemand de nationalité.

Cet individu s'est, en outre, livré au début de la guerre, à des tractations commerciales d'un caractère douteux, et je ne puis en conséquence que maintenir la décision intervenue au sujet de son internement ${ }^{55}$.

Les représailles envers ce sujet allemand s'expliquent par ses activités commerciales avec une entreprise installée aux États-Unis mais appartenant à des sujets allemands ${ }^{56}$. Une lettre écrite en allemand adressée à Rohnstadt le 18 mars 1915 a ainsi attiré l'attention des autorités. L'utilisation d'acronymes et d'abréviations commerciales pouvait laisser l'imagination débordante des autorités passer les bornes de la raison. Quelques jours plus tard, l'administration des douanes met ses personnels sous pression en fournissant le nom de ceux qui ont validé l'entrée de marchandises venant des États-Unis et procède au renvoi des abonnements de Rohnstadt en métropole ${ }^{57}$. Le dossier est ensuite défendu par le gouverneur Duprat qui intercède en faveur de l'Allemand en essayant d'atténuer sa "germanité ". Ce ressortissant allemand serait ainsi né en Russie, à Saint-Pétersbourg, sa femme serait française et, comble de l'ironie, son beau-frère serait colonel dans l'armée française ${ }^{58}$ ! Son comportement durant le conflit montre son attachement patriotique à la France, comme l'illustre la mention de son nom sur une liste de souscription établie pour les blessés de guerre en août $1914^{59}$.

Cette phobie concerne également les ressortissants italiens. Tel est le cas de Michel Orrico, âgé de quarante-six ans et marié à une Réunionnaise, fille d'un brigadier de gendarmerie, depuis de nombreuses années. Entrepreneur dans le cinéma, son honorabilité n'est pas remise en cause mais ses fonctions de vice-consul d'Italie sur l'île laissent présager des

54. Correspondance du gouverneur adressée au ministre des colonies, ADR 1M341.

55. Lettre du ministre des Colonies au député Gasparin non datée (1917 ?), ANOM, FM/SG/ REUc553 d6606.

56. ADR, $1 \mathrm{M} 550$.

57. Correspondance du gouverneur envers les particuliers, 1910-1918, ADR 1M637-638.

58. Correspondance du gouverneur adressée au ministre des colonies, ADR 1M550. Né à Saint-Pétersbourg en 1856, Frédéric Rohnstadt a donc cinquante-huit ans au moment des faits. Il s'est marié dans la colonie avec une des filles de la famille Adam de Villiers avec laquelle il a eu quatre enfants. Installé à La Réunion depuis 1886, il est négociant en vanille. Depuis 1914, il est paralysé du bras et de la jambe gauche à la suite d'une attaque d'apoplexie.

59. Nouveau journal de l'île de la Réunion, 20 août 1914, ADR 1PER52/10. 
menées obscures ${ }^{60}$. Quant à François Labanca, âgé de quarante-huit ans, ce dernier est installé à La Réunion depuis près d'une vingtaine d'années et n'ayant pu trouver de situation stable pour honorer son métier d'horloger, il vit misérablement et a contracté de nombreuses dettes. Aucune procédure judiciaire n'est envisagée à l'encontre de ces trois personnes même si elles subissent durant le conflit une discrète surveillance et se trouvent confrontées à l'infamie des rumeurs.

In fine, les dénonciations et les soupçons d'espionnage visant les Italiens et les Allemands participent à une volonté de politique de défense nationale, qui se traduit dans ce domaine, en métropole tout au moins, par la confiscation systématique des biens ennemis et un durcissement du droit de la nationalité. À son échelle, patriotisme oblige, La Réunion entendait sans doute jouer sa partie, si petite soit-elle. Les spécificités de ce patriotisme réunionnais sont peut-être à trouver dans les sentiments d'abandon et d'éloignement éprouvés par la population. On peut ainsi émettre l'hypothèse que les ressentiments populaires contre les populations d'origine indienne et allemandes sont la conséquence d'un désir refoulé de participer de manière plus active au principe de la défense nationale. Si «le tragique naît du heurt de systèmes de représentations incompatibles entre eux ${ }^{61}$ ", les rumeurs lancées contre les ennemis seraient dans cette optique une revendication indirecte de participation au conflit ${ }^{62}$.

Pierre-Éric FAGEOL Université de La Réunion

60. Sa nomination en qualité de vice-consul d'Italie est pourtant considérée comme un excellent choix par les journalistes du Nouveau journal de l'île de la Réunion, 2 septembre 1914, ADR 1PER52/10.

61. Ory Pascal, La Culture comme aventure. Treize exercices d'histoire culturelle, Paris, Complexe, 2008 , p. 20

62. Cet inconscient collectif renvoie à une crise langagière et explique l'imprévisibilité de l'événement. Comme le rappelle John B. Thomson: "Les individus étant les produits d'histoire particulières qui perdurent à travers les habitus, leurs actions ne sauraient être adéquatement analysées comme le résultat d'un calcul délibéré. Les pratiques doivent bien plutôt être envisagées comme le produit d'une rencontre entre un habitus et un champ, mutuellement "compatibles" ou "conformes" à un degré variable, de telle sorte qu'un individu puisse ne pas savoir comment agir, et les mots venir à lui manquer, dans les circonstances où apparaît un manque de conformité " (in Pierre Bourdieu, Langage et pouvoir symbolique, op. cit., pp. 30-31). 